\title{
Properties of a mixed-valent iron compound with the kagomé lattice
}

\author{
C. N. R. Rao,* Geo Paul, and Amitava Choudhury \\ Chemistry and Physics of Materials Unit and CSIR Centre of Excellence in Chemistry, Jawaharlal Nehru Centre for Advanced Scientific \\ Research, Jakkur P.O., Bangalore 560 064, India \\ E. V. Sampathkumaran \\ Department of Condensed Matter Physics and Materials Science, Tata Institute of Fundamental Research, Mumbai 400005 , India \\ Arup K. Raychaudhuri \\ Department of Physics, Indian Institute of Science, Bangalore 560 012, India \\ S. Ramasesha and Indranil Rudra \\ Solid State and Structural Chemistry Unit, Indian Institute of Science, Bangalore 560 012, India
}

\begin{abstract}
An organically templated iron sulfate of the formula $\left[\mathrm{HN}\left(\mathrm{CH}_{2}\right)_{6} \mathrm{NH}\right]\left[\mathrm{Fe}^{\mathrm{III}} \mathrm{Fe}_{2}^{\mathrm{II}} \mathrm{F}_{6}\left(\mathrm{SO}_{4}\right)_{2}\right] \cdot\left[\mathrm{H}_{3} \mathrm{O}\right]$ possessing the kagomé lattice has been prepared and characterized by single-crystal crystallography and other techniques. This mixed-valent iron compound shows complex magnetic properties including spin-glass behavior and magnetic hysteresis. The low-temperature specific heat data show deviation from the $T^{2}$ behavior found in two-dimensional frustrated systems. Simple calculations have been carried out to understand the properties of this kagomé compound.
\end{abstract}

\section{INTRODUCTION}

Geometrically frustrated magnets constitute an interesting class of materials. Of these, the two-dimensional (2D) compounds possessing the kagomé lattice have attracted considerable attention. ${ }^{1,2}$ There have been several papers on the magnetic and related properties of jarosites possessing the kagomé lattice, but a complete understanding of these materials has been rendered difficult, partly because it is difficult to synthesize pure stochiometric samples. One category of jarosites appears to exhibit long-range antiferromagnetism, while the other has a spin-glass-type behavior, the latter exemplified by the $\left(\mathrm{D}_{3} \mathrm{O}\right)^{+}$salt with $100 \%$ site occupation. ${ }^{2,3}$ All the iron compounds with the kagomé lattice studied to date have $\mathrm{Fe}$ in one spin state (generally $S=5 / 2$ ). To our knowledge, no kagomé iron compound containing iron in the two spin states has been reported. We have been able to prepare an organically templated iron sulfate of the formula $\left[\mathrm{HN}\left(\mathrm{CH}_{2}\right)_{6} \mathrm{NH}\right]\left[\mathrm{Fe}^{\mathrm{III}} \mathrm{Fe}_{2}^{\mathrm{II}} \mathrm{F}_{6}\left(\mathrm{SO}_{4}\right)_{2}\right] \cdot\left[\mathrm{H}_{3} \mathrm{O}\right], \quad$ I, possessing the kagome lattice wherein $\mathrm{Fe}$ is in both $2+$ and $3+$ states. We report the properties of such a kagomé lattice with twospin states of iron.

\section{EXPERIMENT}

The synthesis of the iron sulfate I was carried out in a $23-\mathrm{cm}^{3}$ Teflon-lined acid digestion bomb under autogenous pressure by heating the starting mixture of ferric citrate (1 $\mathrm{mM}), \mathrm{H}_{2} \mathrm{SO}_{4}$ (98\%, $\left.4 \mathrm{mM}\right)$, 1,4-diazabicyclo[222] octane (DABCO, $6 \mathrm{mM}), \mathrm{HF}(48 \%, 4 \mathrm{mM}), \mathrm{H}_{2} \mathrm{O}(50 \mathrm{mM})$, and $n$-butanol $(30 \mathrm{mM})$ at $180{ }^{\circ} \mathrm{C}$ for $48 \mathrm{~h}$. The product consisting of hexagonal-plate-shaped crystal was monophasic and subsequently characterized by single-crystal and powder $\mathrm{x}$-ray diffraction. Single-crystal data were collected on a Siemens SMART-CCD diffractometer (graphitemonochromated Mo $K \alpha$ radiation, $\lambda=0.71073 \AA, \quad T$ $=298 \mathrm{~K}$ ), and the structure determination was carried out by direct method using SHELXS-86 (Ref. 4) and the refinement was carried out using the SHELXTL-PLUS (Ref. 5) package of programs. Crystal data for $\mathbf{I}$ : orthorhombic, space group Pna2 $_{1} \quad$ (No. 33), $a=12.9269(6), \quad b=7.4091(3), \quad c$ $=17.5970(8) \AA, \quad \alpha=\beta=\gamma=90^{\circ}, \quad V=1685.38(13) \AA^{3}, \quad Z$ $=4$, final $R=0.0243, R_{w}=0.0562$, and $\mathrm{GOF}=1.000$. The $\mathrm{X}$-ray powder diffraction pattern was in good agreement with the simulated pattern generated from the single-crystal data, indicating the phase purity of the sample. The magnetic measurements were carried out by using a superconducting quantum interference device (SQUID) magnetometer. Specific heat measurement was carried out by employing a quasiadiabatic ${ }^{6}$ pulse technique.

\section{RESULTS AND DISCUSSION}

The single-crystal x-ray crystallographic study of I clearly shows that the structure consists of anionic layers of cornersharing $\mathrm{Fe}^{\mathrm{III}} \mathrm{F}_{4} \mathrm{O}_{2}$ and $\mathrm{Fe}^{\mathrm{II}} \mathrm{F}_{4} \mathrm{O}_{2}$ octahedra and $\mathrm{SO}_{4}$ tetrahedra. In Fig. 1, we show the structure of the compound to demonstrate how the kagomé lattice is derived from three and six rings of octahedra, giving rise to the hexagonal tungsten-bronze-type sheets. There is $100 \%$ occupation, rendered possible by the presence of fluorine instead of the $\mathrm{OH}$ groups normally found in jarosites. The Fe-O bond distances in $\mathbf{I}$ are in the range $2.003(3)-2.149(3) \AA\left[\left(\mathrm{Fe}^{\mathrm{II}}-\mathrm{O}\right)_{\mathrm{av}}\right.$ $=2.14 \AA$ and $\left.\left(\mathrm{Fe}^{\mathrm{III}}-\mathrm{O}\right)_{\mathrm{av}}=2.011(5) \AA\right]$. The Fe-F bond distances are in the range 1.906(2)-2.157(2) $\left[\left(\mathrm{Fe}^{\mathrm{II}}-\mathrm{F}\right)_{\mathrm{av}}\right.$ $=2.08 \AA$ and $\left.\left(\mathrm{Fe}^{I I I}-\mathrm{F}\right)_{\mathrm{av}}=1.936(2) \AA\right]$. The bond angle values show that all the iron atoms form near-perfect octahedra. Bond valence sum calculations $[\mathrm{Fe}(1)=1.93, \mathrm{Fe}(2)$ 


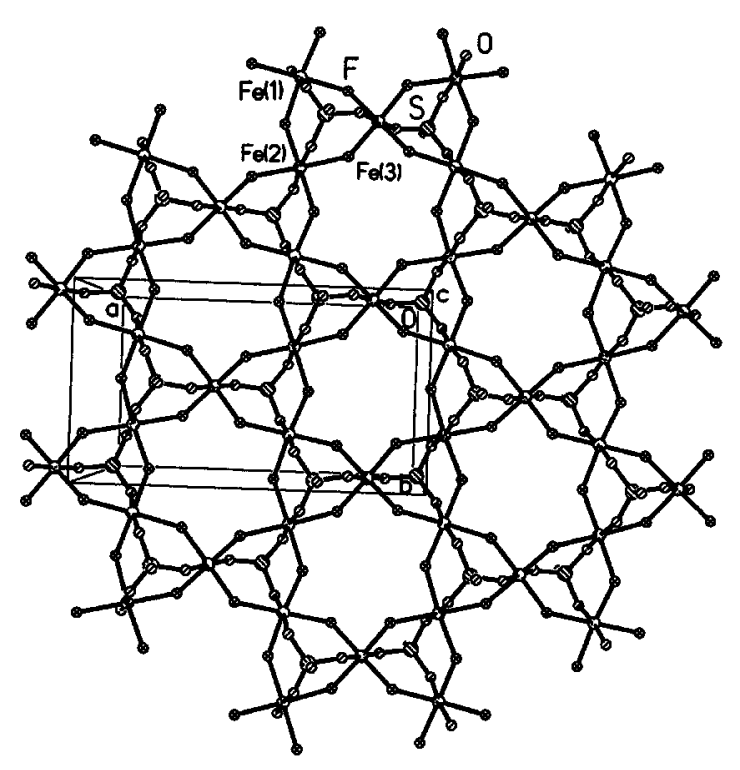

(a)

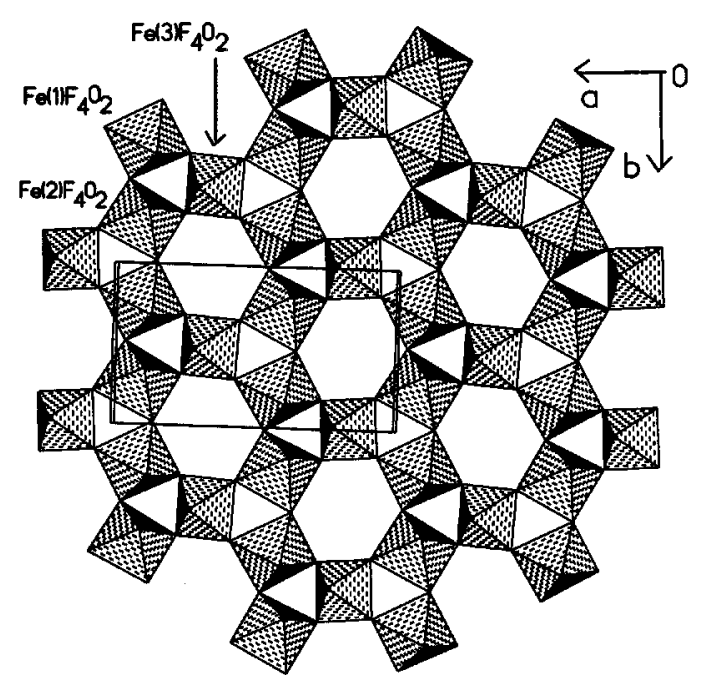

(b)

FIG. 1. (a) Ball and stick representation of a section of $\left[\mathrm{Fe}^{\mathrm{III}} \mathrm{Fe}_{2} \mathrm{II}_{6}\left(\mathrm{SO}_{4}\right)_{2}\right]^{3-}$ in $\mathbf{I}$, showing the presence of the three and six rings, where the three rings are capped by sulfate tetrahedra. (b) Polyhedral view of the 2D network of corner-sharing iron octahedra in $\mathbf{I}$ in the $a b$ plane. Here $\mathrm{Fe}(1)$ and $\mathrm{Fe}(2)$ are in the +2 state and $\mathrm{Fe}(3)$ is in +3 state.

$=1.92$, and $\mathrm{Fe}(3)=3.02]$ as well as the values of the average bond distances indicate the oxidation states of $\mathrm{Fe}(1)$ and $\mathrm{Fe}(2)$ to be +2 and of $\mathrm{Fe}(3)$ to be +3 . The positions of the four bridging $\mathrm{F}$ atoms are also supported by the bond valence calculations $[\mathrm{F}(1)=0.734, \mathrm{~F}(2)=0.669, \mathrm{~F}(3)=0.797, \mathrm{~F}(4)$ $=0.70, F(5)=0.76$, and $F(6)=0.71]$. The ${ }^{57} \mathrm{Fe}$ Mössbauer spectrum of the compound showed the presence of the $\mathrm{Fe}^{\mathrm{II}}$ and $\mathrm{Fe}^{\mathrm{III}}$ states in the ratio $2: 1$, consistent with the formula derived from crystallography and chemical analysis.

The magnetic properties of the mixed-valent kagomé compound I are complex indeed. In Fig. 2(a), we show the dc magnetic susceptibility data under field-cooled (FC) and zero-field-cooled (ZFC) conditions. The observed divergence between the FC and ZFC data is characteristic of a magnetically frustrated system, with a spin-freezing temperature $\left(T_{f}\right)$ of $\sim 13 \mathrm{~K}$. ac susceptibility measurements [Fig. 2(b)]
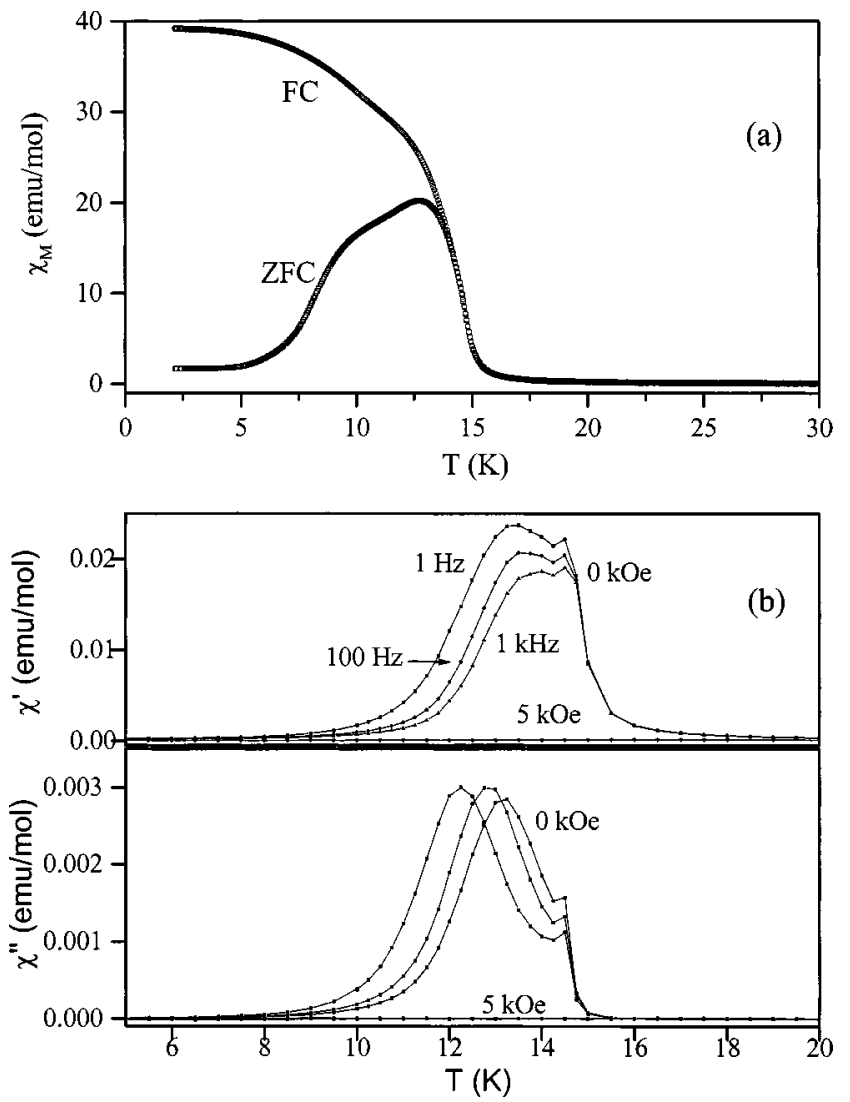

FIG. 2. (a) dc susceptibility data of I showing the divergence between the ZFC and FC behavior in the presence of a magnetic field of 100 Oe. (b) Frequency dependence of ac susceptibility data ( $\chi^{\prime \prime}$ out-of-phase component) shows the characteristic spin-glass behavior.

confirm the spin-glass nature of $\mathbf{I}$. The frequency dependence of the ac susceptibility in zero field disappears at a field of 5 kOe, confirming the spin-glass behavior. In Fig. 3(a), we show the temperature variation of the inverse dc magnetic susceptibility measured at $5 \mathrm{kOe}$. The high-temperature data are linear, extrapolating to a large negative Curie temperature $(-180 \mathrm{~K})$. The isothermal $(2.2 \mathrm{~K})$ magnetization data at an applied field of $12 \mathrm{~T}$ give a moment $2.85 \mu_{B} / \mathrm{mol}$, but we observe a transition to a magnetic state at low temperatures [Fig. 3(a)]. Interestingly, we do observe magnetic hysteresis at $10 \mathrm{~K}$ as shown in Fig. 3(b). The magnetic hysteresis data of I show a steplike behavior and a metamagnetic transition, the field required for the transition depending on the temperature. We show the hysteresis curve at $2.2 \mathrm{~K}$ in Fig. 4 to illustrate these features. We believe I to be the first example of an iron compound with the kagomé lattice exhibiting such a magnetic behavior. We are not in a position to fully interpret the magnetic properties of $\mathbf{I}$, in the absence of the magnetic structure. What is important to note, however, is the marked difference in the magnetic behavior of $\mathbf{I}$ from that of other kagomé compounds with $\mathrm{Fe}$ in a single oxidation state.

Specific heat measurements on the mixed-valent kagomé compound $\mathbf{I}$ in the $3-20 \mathrm{~K}$ range have yielded interesting results. The specific heat shows a broad peak in the region of $T_{f}$ (Fig. 5), somewhat comparable to that in a kagomé spin 

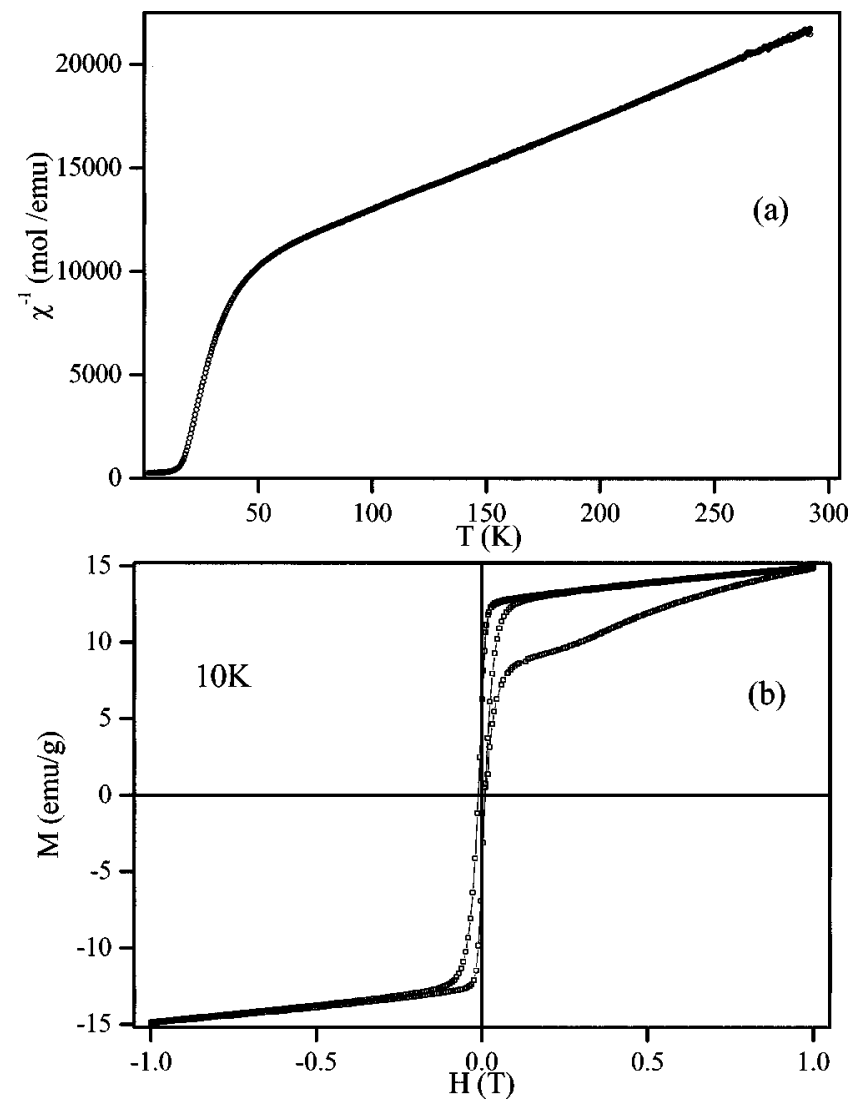

FIG. 3. (a) Temperature variation of the inverse dc susceptibility measured at $5 \mathrm{kOe}$. (b) Magnetic hysteresis data of $\mathbf{I}$ at $10 \mathrm{~K}$.

glass. $^{7}$ A plot of $C$ versus $T$ shows the absence of a $\lambda$ anomaly at the transition and instead shows a shoulder as expected of spin-glass ordering. This behavior is, however, different from the $\mathrm{D}_{3} \mathrm{O}^{+}$jarosite ${ }^{8}$ where there is only a slope change in the heat capacity at the magnetic transition. The hump around $7 \mathrm{~K}$ is an artifact arising from the presence of the peak around $15 \mathrm{~K}$ and the unusual low-temperature behavior. The low-temperature behavior (Fig. 5) does not conform to the simple $T^{2}$ dependence exhibited by kagomé Fe compounds. $^{7,8}$ The data below $10 \mathrm{~K}$ can be fitted into the

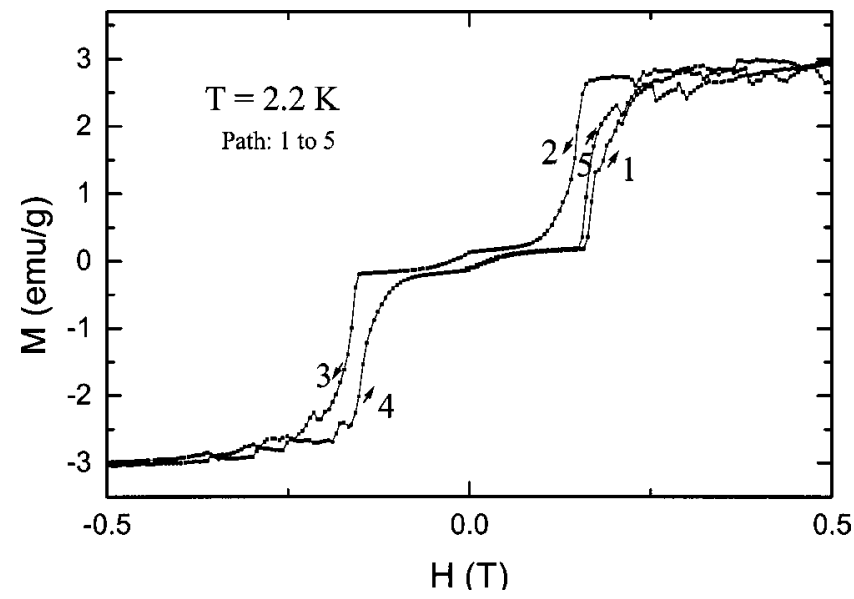

FIG. 4. Hysteresis data at $2.2 \mathrm{~K}$ showing transitions.

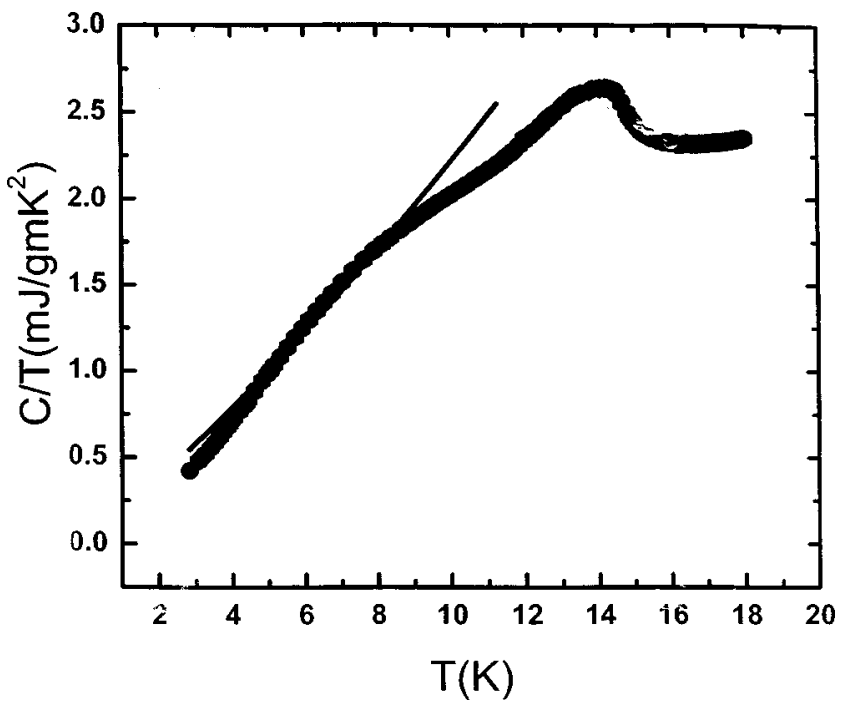

FIG. 5. Specific heat data in the $3-20 \mathrm{~K}$ range.

relation $C=\alpha T e^{(-\gamma / T)}+\beta T^{2}+\delta T^{3}$, with a small contribution from the first and third terms. The fit shown in Fig. 5 has $\alpha=17 \mathrm{~mJ} \mathrm{~mol}^{-1} \mathrm{~K}^{2}, \quad \beta=128 \mathrm{~mJ} \mathrm{~mol}^{-1} \mathrm{~K}^{3}, \quad$ and $\delta$ $=2.98 \mathrm{~mJ} \mathrm{~mol}^{-1} \mathrm{~K}^{4}$. The value of $\alpha$ is about a factor 2 higher and $\delta$ about a factor of 10 higher than in the rare-earth manganates which are ferromagnetic metals. ${ }^{9}$ Part of the enhancement of the linear as well as the cubic terms can arise from excitations in linear and 3D antiferromagnetic chains. The gap implies that linear excitations are damped. A linear term can also come from a two-level system.

In order to understand the nature of the mixed-valent kagomé lattice, we have carried out some calculations using the nearest-neighbor antiferromagnetic exchange Hamiltonian to model the mixed kagomé clusters, wherein we have assumed a cyclic boundary condition with all the exchange constants antiferromagnetic and equal. The geometry and interactions of the spins in the cluster are shown in Fig. 6. The $M_{s}=0$ Hilbert space can be partitioned into smaller evenand odd-parity subspaces of $\sim 37 \times 10^{6}$ by exploiting parity (invariance of the Hamiltonian to flipping all spins about the $z$ axis). However, even this is too large for exact computation

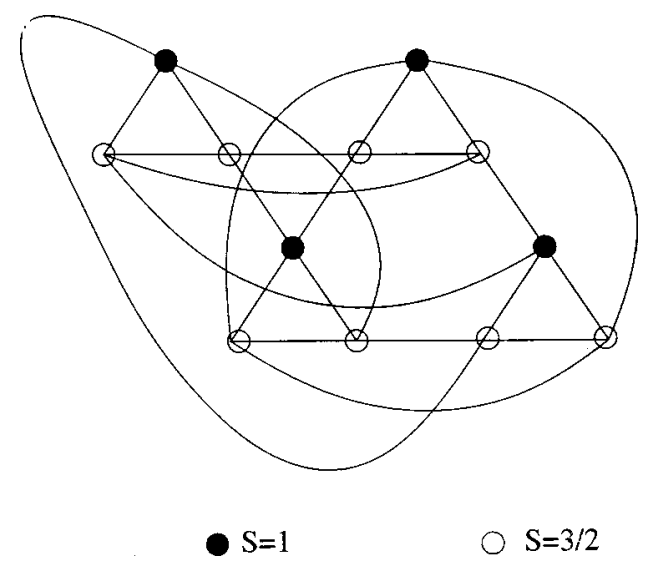

FIG. 6. Twelve-site kagomé cluster with periodic boundary condition. 


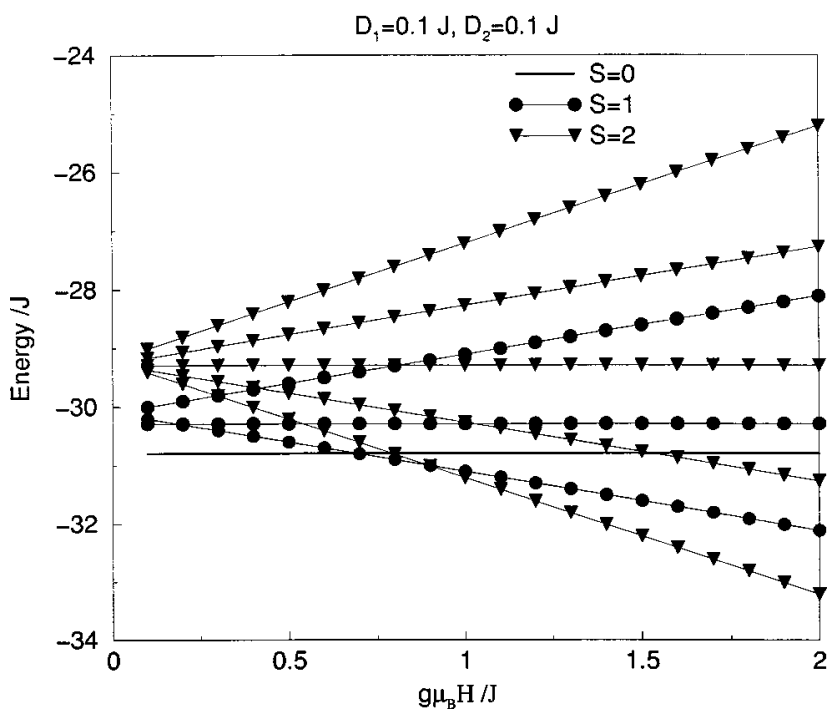

FIG. 7. Spin-state crossing for $\mathbf{I}$ with change in magnetic field.

of the eigenstates and their properties. To reduce the dimensionality of the spaces, therefore, and yet retain the essential features of the mixed-spin kagomé lattice, we have modeled a spin-3/2-spin-1 system. This system has the essential features of the spin-5/2-spin-2 kagomé lattice. One of the spins is integral, while the other half odd-integral and both allow for intra-ion dipolar interactions. The dipolar interaction within each ion leads to the dipolar Hamiltonian $\hat{\mathbf{H}}^{\prime}$ is given by

$$
\hat{\mathbf{H}}^{\prime}=D_{1} \sum_{i \in N_{1}}\left(\hat{\mathbf{s}}_{i}^{z}\right)^{2}+D_{2} \sum_{i \in N_{2}}\left(\hat{\mathbf{s}}_{i}^{z}\right)^{2},
$$

where $D_{1}$ and $D_{2}$ are the anisotropy constants for two different ions and $N_{1}$ and $N_{2}$ are the number of these ions in the system. Usually, dipolar interactions are small compared to the exchange interactions. Hence we treat $\hat{\mathbf{H}}^{\prime}$ as a perturbation over the eigenstates of $\hat{\mathbf{H}}_{0}$ and compute energies correct to first order in $\hat{\mathbf{H}}^{\prime}$.

The ground state of $\hat{H}_{0}$ is a singlet, and the first excited state, also a singlet, is $0.58 \mathrm{~J}$ above the ground state. The single-ion anisotropy alters this energy level ordering. The singlet-singlet gap is unaffected by $\hat{H}^{\prime}$. In the case of the singlet-triplet gap, the triplet state becomes the ground state at $D_{1}=0.01 \mathrm{~J}$ and $D_{2}=0.4 \mathrm{~J}$. Here $D_{2}$ has a stronger effect than $D_{1}$ in switching the ground state from singlet to triplet, since $\Sigma_{i}\left\langle\hat{\mathbf{s}}_{i}^{z}\right\rangle$ will always have more weight for the eight spin$3 / 2$ objects than the four spin-1 objects in the cluster. For large enough $D_{1}$ and $D_{2}$ values, the ground state switches to a quintet.

To study the dependence of magnetization on the magnetic field, we introduce a Zeeman term along with the single-ion anisotropy and investigate the ground-state spin with increasing magnetic field at various fixed values of $D_{1}$ and $D_{2}$. We consider only the ground-state singlet, the low-

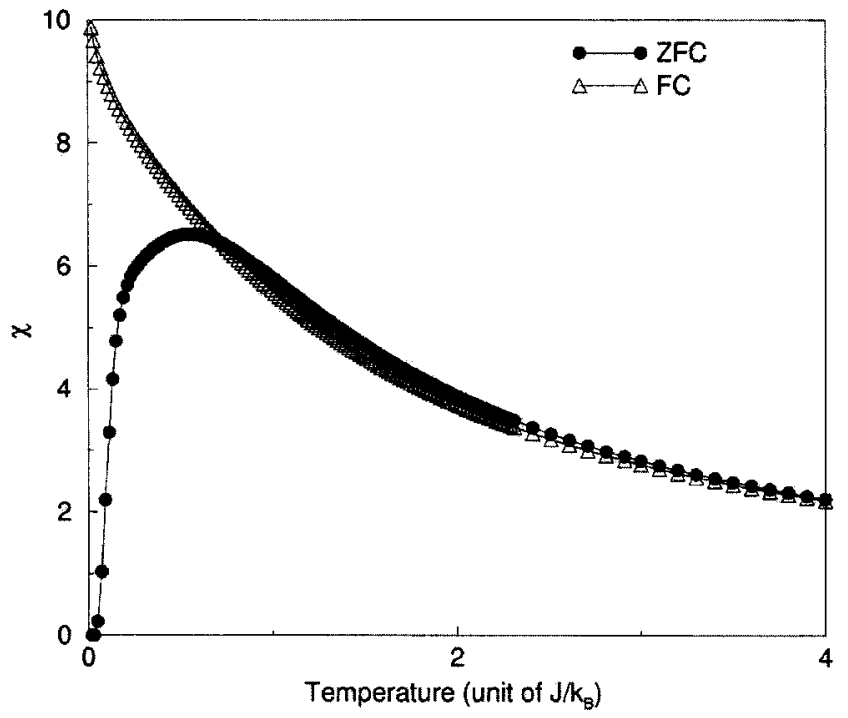

FIG. 8. Divergence in the ZFC and FC behavior calculated by using a few low-lying states.

est triplet and lowest quintet states in this study. From Fig. 7, we find that when $D_{1}=0.1 \mathrm{~J}$ and $D_{2}=0.1 \mathrm{~J}$ at low magnetic fields, the ground state is the singlet. As the magnetic field is increased, the ground state switches to a triplet and at still higher fields the quintet becomes the ground state. A similar crossover is observed for other values of $D_{1}$ and $D_{2}$. The values of $D_{1}$ and $D_{2}$ determine the nature of the ground state and the critical fields for spin-state crossovers. The crossover of spin states predicts a steplike behavior of the magnetization, with increasing magnetic field at low temperatures, which appears to be the case as indicated by the measurements at $2.2 \mathrm{~K}$ shown in Fig. 4.

We have examined the low-temperature FC and ZFC susceptibility behavior by obtaining lowest six eigenstates in $M_{s}=0$ to \pm 5 subspaces. We have calculated the Boltzmann factor for these low-lying states and obtained the ZFC and FC susceptibilities as functions of temperature for fixed values of the single-ion anisotropy constants $\left(D_{1}=0.1 \mathrm{~J}\right.$ and $D_{2}=0.1 \mathrm{~J}$ ). The ZFC and FC susceptibilities are calculated as derivatives of the magnetization with respect to the magnetic field at zero magnetic field and at a magnetic field of $0.5 \mathrm{~J} / \mu_{B}$. We are able to reproduce the divergence behavior of the ZFC and FC susceptibilities at low temperatures found experimentally as can be seen from Fig. 8. Our model calculations predict the low-temperature specific heat data which are best fitted with a relation $C=\alpha+\beta T+\gamma T^{2}+\delta T^{3}$, with the $T^{2}$ term being the most dominant. Clearly, more detailed investigations are required to fully understand the magnetic properties and specific heat behavior of this mixed-valent $\mathrm{Fe}$ compound with the kagomé lattice.

\section{ACKNOWLEDGMENT}

The authors thank BRNS (DAE), India, for support of this research. 
*Electronic address: cnrrao@jncasr.ac.in

${ }^{1}$ A. P. Ramirez, Annu. Rev. Mater. Sci. 24, 453 (1994).

${ }^{2}$ J. E. Greedan, J. Mater. Chem. 11, 37 (2001).

${ }^{3}$ A. S. Wills, A. Harrison, C. Ritter, and R. I. Smith, Phys. Rev. B 61, 6156 (2000).

${ }^{4}$ G. M. Sheldrick, SHELXS-86, computer program for crystal structure determination, University of Göttingen, Göttingen, Germany, 1986.

${ }^{5}$ G. M. Sheldrick, SHELXTL-PLUS, computer program for crystal structure solution and refinement, University of Göttingen,
Göttingen, Germany, 1993

${ }^{6}$ S. Banerjee, M. W. J. Prins, K. J. Rajeev, and A. K. Raychaudhuri, Pramana, J. Phys. 39, 391 (1992).

${ }^{7}$ A. P. Ramirez, C. P. Espinosa, and A. S. Cooper, Phys. Rev. B 45, 2505 (1992).

${ }^{8}$ A. S. Wills, A. Harrison, S. A. M. Mentink, T. E. Mason, and Z. Tun, Europhys. Lett. 42, 325 (1998).

${ }^{9}$ J. J. Hamilton, E. L. Keatly, H. L. Ju, A. K. Raychaudhri, V. N. Smolyaminova, and R. L. Greene, Phys. Rev. B 54, 14926 (1996). 